\title{
Removal of calcific band keratopathy without ethylenediaminetetraacetic acid (EDTA) in eyes with limited visual potential
}

This article was published in the following Dove Press journal:

Clinical Ophthalmology

\author{
Carson R Bee' \\ Lisa R Koenig 2 \\ Eileen S Hwang' \\ Steven B Koenig' \\ 'Department of Ophthalmology, \\ Froedtert and the Medical College of \\ Wisconsin, Milwaukee, WI, USA; ${ }^{2}$ Weill \\ Cornell Medical College, New York, \\ NY, USA
}

\begin{abstract}
Purpose: Treatment of calcific band keratopathy (CBK) is commonly performed with ethylenediaminetetraacetic acid (EDTA), but EDTA has become more difficult to obtain. This paper describes a technique for treating CBK using a diamond-dusted burr without EDTA in eyes with limited visual potential.
\end{abstract}

Patients and methods: In this paper, we provide detailed instructions on how to perform the surgical technique for treating CBK, along with a surgical video. We performed a retrospective review of consecutive patients with clinically significant $\mathrm{CBK}$ who underwent this procedure from December 2012 to July 2015.

Results: Seven eyes of six patients were included for analysis. Preoperatively, all patients suffered from ocular discomfort. All eyes had poor preoperative visual acuity due to non-corneal ocular disease. The most common causes of CBK in this series were retinopathy of prematurity (three eyes) and chronic uveitis (two eyes). Postoperatively, all patients reported partial or complete relief of discomfort. The length of follow-up ranged from 15 days to 31 months. Two eyes experienced recurrence of CBK. This occurred at 4 and 28 months after treatment.

Conclusion: The diamond-dusted burr can easily and effectively remove the corneal epithelium and underlying calcium deposits. Therefore, it may be used to effectively treat discomfort from CBK. Keywords: diamond-dusted burr, superficial keratectomy, corneal epithelial debridement, chelation

\section{Introduction}

In calcific band keratopathy (CBK), calcium accumulates in the superficial layers of the cornea in the interpalpebral fissure zone, most often due to chronic ocular inflammation or systemic hypercalcemia. ${ }^{1,2}$ The calcium deposits cause pain, foreign body sensation, and decreased visual acuity. The most common methods for removing the calcium deposits are mechanical debridement with a blade, chemical chelation with ethylenediaminetetraacetic acid (EDTA), and phototherapeutic keratectomy.

Manual debridement of CBK by scraping with a blade is effective but can lead to an irregular corneal surface. ${ }^{3,4}$ Chelation with EDTA works by sequestering calcium and softening the deposits. Subsequent mechanical debridement is often required, but this method results in a smoother corneal surface. Chelation with EDTA is currently the most popular treatment for CBK, but EDTA recently ceased to be commercially available in the US. Another option for CBK treatment is phototherapeutic keratectomy, in which the superficial cornea is ablated with an excimer laser, but this method is costly and can cause refractive shifts. Even so, this method may be preferred for eyes with good visual potential. In this paper, we present a method for debridement of CBK with
Correspondence: Steven B Koenis Froedtert and the Medical College of Wisconsin, 925 North 87th Street, Milwaukee, WI 53226, USA

Tel +l $4 \mid 49552020$

Fax +l 4149556300

Email skoenig@mcw.edu 
a diamond-dusted burr without EDTA. We provide the results of seven consecutive eyes with limited visual potential treated by this simple, quick, and inexpensive technique.

\section{Methods}

\section{Chart review}

A retrospective chart review was conducted in accordance with the tenets of the Declaration of Helsinki. A waiver of informed consent and the study were approved by the Institutional Review Board of Medical College of Wisconsin. Using a computerized database, we searched for deidentified records of patients with a diagnosis of CBK who were evaluated at the Froedtert Eye Institute, Milwaukee, WI, between December 2012 and July 2015. We identified seven consecutive eyes that underwent CBK treatment with a diamond-dusted burr without EDTA. The procedure was performed by a single surgeon (SBK) using the standard technique as described below. Visual acuities were noted at the clinic visit preceding surgery (preoperative visual acuity) and at the last clinic visit (final visual acuity). Patients were noted to have symptomatic relief if they described improved eye comfort at any postoperative clinic visit. Recurrence was defined as increased calcium deposits on slit lamp examination by the surgeon.

\section{Results}

\section{Description of surgical technique}

The procedure may be performed at a slit lamp or in a minor surgical suite using a portable microscope under topical anesthesia with $0.5 \%$ proparacaine. The eye is prepped with $10 \%$ povidone iodine and draped with a small pre-cut aperture plastic drape (style 1020; 3M, Maplewood, MN,

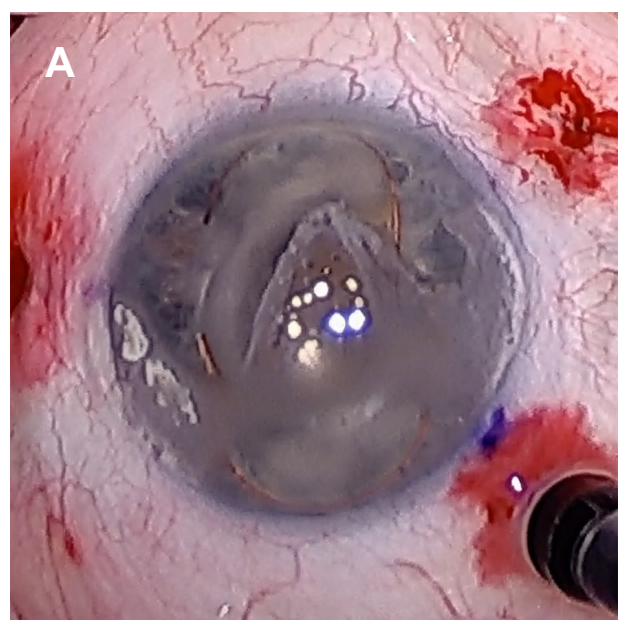

Figure I Appearance before (A) and after (B) treatment of calcific band keratopathy with a diamond-dusted burr without ethylenediaminetetraacetic acid.

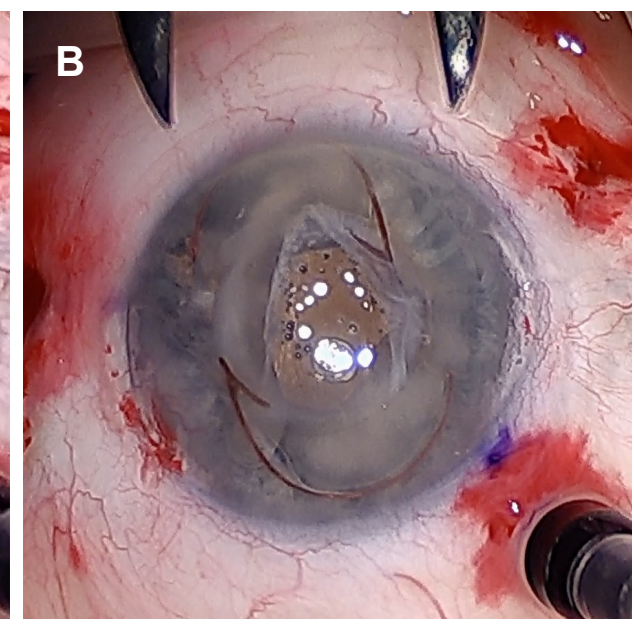

USA). After placing a Nevyas Drape Retractor (Wilson Ophthalmics, Mustang, OK, USA), a thin wire Barraquer speculum is used to separate the lids. In eyes with markedly elevated calcific plaques, a \#69 Beaver blade (Beaver Visitec, Waltham, MA, USA) may be used first to debulk the calcific deposits. This blade may also be used to debride the corneal epithelium overlying the calcific band, although this is not necessary. Using the battery-powered reusable $5.0 \mathrm{~mm}$ diamond-dusted burr (Ophtho-Burr ${ }^{\circledR}$; Beaver Visitec), the surgeon applies gentle and even pressure to the cornea in either a rotary or a back-and-forth manner until the calcium is removed (Figure 1 and Video S1). It is important that excessive pressure is not applied to a single location to avoid creating a thin area and to "feather" the edges of the treatment zone to provide a gradual transition to healthy cornea. The cornea is lubricated with balanced salt solution at about one drop per second during the debridement. The debridement using the burr takes about 5 minutes. The remaining corneal epithelial defect is managed with a bandage soft contact lens, and Maxitrol ointment (neosporin, polymyxin B, and dexamethasone; Alcon, Fort Worth, TX, USA) is applied four times daily for 1 week. The bandage soft contact lens is typically removed after 1 week, and patients are treated with topical prednisolone acetate $1 \%$ drops tapered over a month.

\section{Patient characteristics}

Seven eyes from six patients were included for analysis (Table 1). Preoperatively, all patients suffered from discomfort in the eye that underwent the procedure. All eyes had poor preoperative visual acuity due to non-corneal ocular disease. The average age was 22 years. The most common 
Table I Preoperative and postoperative characteristics of eyes that underwent treatment of calcific band keratopathy with a diamonddusted burr without EDTA

\begin{tabular}{|l|l|l|l|l|l|l|l|l|}
\hline Subject & $\begin{array}{l}\text { Age } \\
\text { (years) }\end{array}$ & Sex & Etiology & $\begin{array}{l}\text { Preoperative } \\
\text { visual acuity }\end{array}$ & $\begin{array}{l}\text { Final visual } \\
\text { acuity }\end{array}$ & $\begin{array}{l}\text { Follow-up } \\
\text { (months) }\end{array}$ & $\begin{array}{l}\text { Symptomatic } \\
\text { relief }\end{array}$ & Recurrence \\
\hline I & 5I & M & Proliferative diabetic retinopathy & NLP & NLP & 30 & Yes & Yes, at 28 months \\
\hline 2 & 33 & F & Chronic uveitis & $20 / 200$ & HM & II & Yes & Yes, at 4 months \\
\hline 3 & II & M & Retinopathy of prematurity & HM & CF & 8 & Yes & No \\
\hline 4 & II & M & Retinopathy of prematurity & LP & LP & I & Yes & No \\
\hline 5 & I0 & M & Chronic uveitis & $20 / 200$ & $20 / 400$ & 16 & Yes & No \\
\hline 6 & I9 & M & Retinopathy of prematurity & 20/200 & $20 / 400$ & 0.5 & Yes & No \\
\hline 7 & I5 & M & Acute retinal necrosis & LP & LP & 16 & Yes & No \\
\hline
\end{tabular}

Abbreviations: EDTA, ethylenediaminetetraacetic acid; M, male; F, female; NLP, no light perception; HM, hand motion; CF, count fingers; LP, light perception.

causes of CBK in this series were retinopathy of prematurity (three eyes) followed by chronic uveitis (two eyes). Postoperatively, all the patients reported partial or complete symptomatic relief.

\section{Postoperative results}

The length of follow-up ranged from 15 days to 31 months (mean 12 months). At the last available follow-up visit, visual acuity improved in one eye, remained the same in three eyes, and worsened in three eyes. The primary cause of decreased visual acuity in all three cases was progression of non-corneal ocular disease. No complications occurred during surgery, and no complications were noted during long-term follow-up. Two eyes experienced recurrence of CBK. The recurrence episodes occurred at 4 and 28 months after treatment.

\section{Discussion}

CBK results from the deposition of calcium salts in the Bowman's layer and superficial corneal stroma. ${ }^{1}$ Opacification of the central cornea causes loss of vision and glare, while elevated calcific plaques may contribute to pain, foreign body sensation, photophobia, and tearing. ${ }^{2}$

The current options for treatment of CBK include scraping with a blade, chelation with EDTA, and phototherapeutic keratectomy. However, each of these methods has limitations. One major new limitation is that EDTA is no longer commercially available in the US, so surgeons must acquire EDTA from a compounding pharmacy.

This paper describes the use of a hand-held batterypowered diamond-dusted burr to treat $\mathrm{CBK}$ without using EDTA. Bokosky et al described treating CBK using a Fisch power-driven drill with a $5 \mathrm{~mm}$ diamond-dusted tip together with EDTA chelation. ${ }^{5}$ McGrath and Lee described debridement with a blade, needle, microsponge, and/or diamonddusted burr without using EDTA for a variety of conditions, including band keratopathy. ${ }^{6}$ We confirm McGrath and Lee's findings that EDTA is not needed to treat band keratopathy, and specifically highlight the use of the diamond-dusted burr. The diamond-dusted burr is inexpensive, highly portable, and unlikely to cause significant stromal thinning or perforation. We describe seven cases of successful relief of pain and foreign body sensation due to extensive $\mathrm{CBK}$ in eyes with poor visual potential. In these eyes, there is no expectation of improved vision, minimal concern about stromal thinning, and no reliable means of assessing preoperative topography or pachymetry due to the dense, elevated calcium deposits. For eyes with good visual potential, phototherapeutic keratectomy may be the best alternative to EDTA for optimal refractive outcomes.

In patients with elevated calcific plaques, the burr leaves a polished surface, with reduced pain and foreign body sensation. Postoperatively, patients receive a bandage soft contact lens and a combination antibiotic-steroid ointment to reduce foreign body sensation, prevent infection, and promote reepithelialization. Reepithelialization usually occurs within 1-2 weeks, depending upon the size of the residual epithelial defect. Although postoperative infection is a risk of this procedure, there were no cases of infectious keratitis in this series. Two patients experienced recurrence of CBK, which is a known outcome in all the methods of removal. This figure is in line with a recent series that described recurrence in $28 \%$ of patients after chelation with EDTA. ${ }^{7}$ The recurrence interval in our small series treated without EDTA (4 and 28 months) was also grossly similar to the recurrence interval in the large series of patients treated with EDTA (19 months). ${ }^{7}$ Our practice is to counsel all patients preoperatively that CBK is likely to recur and may require periodic retreatment.

\section{Conclusion}

A hand-held battery-powered diamond-dusted burr may be used to remove the calcium deposits without the use of 
blade or EDTA in patients with CBK. Since EDTA is no longer commercially available, this technique may be useful for eyes with poor visual potential. Future studies are needed with a larger sample size to determine whether this technique effectively relieves discomfort and to assess rates of complications and recurrences.

\section{Acknowledgments}

Source of funding: this work was supported by a gift from Doctor Myrna Larson and Doctor Nelson A Moffat and Research to Prevent Blindness.

\section{Disclosure}

The authors report no conflicts of interest in this work.

\section{References}

1. Jhanji V, Rapuano CJ, Vajpayee RB. Corneal calcific band keratopathy. Curr Opin Ophthalmol. 2011;22(4):283-289.

2. Najjar DM, Cohen EJ, Rapuano CJ, Laibson PR. EDTA chelation for calcific band keratopathy: results and long-term follow-up. Am J Ophthalmol. 2004;137(6):1056-1064.

3. Wood TO, Walker GG. Treatment of Band Keratopathy. Am JOphthalmol. 1975;80(3):553.

4. Grant WM. New treatment for calcific corneal opacities. AMA Arch Ophthalmol. 1952;48(6):681-685.

5. Bokosky JE, Meyer RF, Sugar A. Surgical treatment of calcific band keratopathy. Ophthalmic Surg. 1985;16(10):645-647.

6. McGrath LA, Lee GA. Corneal epithelial debridement for diagnosis and therapy of ocular surface disease. Clin Exp Optom. 2015;98(2): 155-159.

7. Al-Hity A, Ramaesh K, Lockington D. EDTA chelation for symptomatic band keratopathy: results and recurrence. Eye. 2018;32(1):26-31. 


\section{Supplementary material}

Video SI Video demonstrating the technique for treatment of calcific band keratopathy with a diamond-dusted burr without ethylenediaminetetraacetic acid.

\section{Publish your work in this journal}

Clinical Ophthalmology is an international, peer-reviewed journal covering all subspecialties within ophthalmology. Key topics include: Optometry; Visual science; Pharmacology and drug therapy in eye diseases; Basic Sciences; Primary and Secondary eye care; Patien Safety and Quality of Care Improvements. This journal is indexed on Submit your manuscript here: http://www.dovepress.com/clinical-ophthalmology-journal

\section{Dovepress}

PubMed Central and CAS, and is the official journal of The Society of Clinical Ophthalmology (SCO). The manuscript management system is completely online and includes a very quick and fair peer-review system, which is all easy to use. Visit http://www.dovepress.com/ testimonials.php to read real quotes from published authors. 